\title{
ViralPlaque: a Fiji macro for automated assessment of viral plaque statistics
}

\author{
Marco Cacciabue ${ }^{\text {Corresp., } 1,2}$, Anabella Currá ${ }^{1,2}$, Maria I Gismondi ${ }^{\text {Corresp. 1, } 2}$ \\ 1 Instituto de Agrobiotecnología y Biología Molecular (IABiMo), Instituto Nacional de Tecnología Agropecuaria (INTA), Consejo Nacional de Investigaciones \\ Científicas y Técnicas (CONICET), Hurlingham, Buenos Aires, Argentina \\ 2 Departamento de Ciencias Básicas, Universidad Nacional de Luján, Luján, Buenos Aires, Argentina \\ Corresponding Authors: Marco Cacciabue, Maria I Gismondi \\ Email address: cacciabue.marco@inta.gob.ar, gismondi.maria@inta.gob.ar
}

Plaque assay has been used for a long time to determine infectious titers and characterize prokaryotic and eukaryotic viruses forming plaques. Indeed, plaque morphology and dimensions can provide information regarding the replication kinetics and the virulence of a particular virus. In this work, we present ViralPlaque, a fast, open-source and versatile Image macro for the automated determination of viral plaque dimensions from digital images. Also, a machine learning plugin is integrated in the analysis algorithm for adaptation of ViralPlaque to the user's needs and experimental conditions. A high correlation between manual and automated measurements of plaque dimensions was demonstrated. This macro will facilitate reliable and reproducible characterization of cytolytic viruses with an increased processing speed. 


\section{ViralPlaque: a Fiji macro for automated assessment of viral plaque statistics}

2

3 Marco Cacciabue, Anabella Currá, María Inés Gismondi

4

5 Instituto de Agrobiotecnología y Biología Molecular (IABiMo), Instituto Nacional de

6 Tecnología Agropecuaria (INTA), Consejo Nacional de Investigaciones Científicas y Técnicas

7 (CONICET), Hurlingham, Buenos Aires, Argentina

8

9 Departamento de Ciencias Básicas, Universidad Nacional de Luján, Luján, Buenos Aires, 10 Argentina.

11

12

13 Corresponding Authors:

14 Marco Cacciabue, María Inés Gismondi

15 De los Reseros y N. Repetto s/n, Hurlingham, Buenos Aires, 1686, Argentina

16 E-mail addresses: cacciabue.marco@inta.gob.ar, gismondi.maria@inta.gob.ar 
18 Abstract

19 Plaque assay has been used for a long time to determine infectious titers and characterize

20 prokaryotic and eukaryotic viruses forming plaques. Indeed, plaque morphology and dimensions

21 can provide information regarding the replication kinetics and the virulence of a particular virus.

22 In this work, we present ViralPlaque, a fast, open-source and versatile ImageJ macro for the

23 automated determination of viral plaque dimensions from digital images. Also, a machine

24 learning plugin is integrated in the analysis algorithm for adaptation of ViralPlaque to the user's

25 needs and experimental conditions. A high correlation between manual and automated

26 measurements of plaque dimensions was demonstrated. This macro will facilitate reliable and

27 reproducible characterization of cytolytic viruses with an increased processing speed.

\section{Keywords}

30 ImageJ macro; FIJI; machine learning; plaque assay; plaque size; cytopathic effect; lytic virus

\section{Introduction}

35 Plaque assay is a typical test originally used for bacteriophage characterization and subsequently 36 adapted to eukaryotic viruses to estimate infectious titers or to perform clonal purification of

37 viral agents (d'Hérelle and Smith, 1926; Dulbecco, 1952). Beyond these uses, formation of viral

38 plaques is a relevant phenotypic feature that can withhold essential information of the virus

39 under study. Indeed, plaque statistics (size, clarity, border definition and distribution) can

40 provide important information on the replication kinetics and virulence factors of a virus. 
41 Morphology of plaques has been reported to help distinguish viral isolates and is currently used

42 as an indicator of virus attenuation (Tajima et al., 2009; Goh et al., 2016; Kato et al., 2017; Fan

43 et al., 2018; Moser et al., 2018; Schade-Weskott, van Schalkwyk \& Koekemoer, 2018).

44 Basically, during a plaque assay, a monolayer of susceptible cells is exposed to a serially diluted

45 lytic virus (commonly 5-100 virions per well). An immobilizing overlay (typically agarose or

46 methyl cellulose) is used to prevent uncontrolled viral spreading through the liquid medium.

47 Following initial infection, regions of dead cells are formed as viral replication cycles unfold,

48 forming individual plaques (Baer \& Kehn-Hall, 2014). Typically, cellular monolayers are then

49 fixed and counterstained with neutral red or crystal violet solutions, which facilitates the

50 identification of the plaques formed. After these steps, plaque statistics (e.g. plaque number or

51 dimensions) can be obtained from the infected cellular monolayer by direct visualization and

52 manual recording. Of note, plaque morphology can vary depending on growth conditions and

53 between viral species (Baer \& Kehn-Hall, 2014).

54 Although manual determination of plaque number can be highly sensitive (i.e., it is possible to

55 detect all plaques in an assay independently of their diameter and shape), count and size

56 measurements of viral plaques is laborious, tedious and time consuming. Moreover, the

57 repetitive nature of this procedure can lead to an increase in error rate. In this sense, in the past

58 decades, improved accessibility of scanner and digital cameras has facilitated the automation of

59 the image analysis step, improving speed and objectivity (Choudhry, 2016). However, the

60 majority of software available either focuses on viral quantification (e.g. UFP/ml determination)

61 (Sullivan et al., 2012) or needs specific experimental conditions (e.g. fluorescence microscopy)

62 (Yakimovich et al., 2015; Culley et al., 2016; Katzelnick et al., 2018). Apart from that, in recent

63 years other software has been developed for acquisition of particle size statistics and 
64 quantification of particles from a broad range of assays such as apoptosis in cultured cells 65 (Helmy \& Azim, 2012), clonogenic assays (Cai et al., 2011) and counting of cell, bacterial and 66 yeast colonies and tumor spheroid particles (Geissmann, 2013; Choudhry, 2016). Nonetheless, 67 these programs have been designed for specific assays with different image conditions and are 68 not fit to accurately characterize plaques of viral origin.

69 We developed ViralPlaque, a versatile ImageJ macro for automated detection and analysis of 70 viral plaques. We demonstrate that this method is fast, accurate and suitable on images obtained 71 from different sources such as a cell phone camera or a flatbed scanner. It is tunable in several 72 parameters, like size of plaques and measurements to perform. Lastly, adaptation of ViralPlaque 73 to the user's particular experimental conditions is incorporated through a machine-learning 74 plugin.

\section{Material and methods}

77

78 Plaque assay

79 Plaque assays were performed on baby hamster kidney cells (BHK-21 clone 13; ATCC CCL10) 80 and on African green monkey kidney cells (Vero, ATCC CCL81) as previously described

81 (García Núñez et al., 2010). Cells were maintained at $37{ }^{\circ} \mathrm{C}$ and $5 \% \mathrm{CO}_{2}$ in Dulbecco's modified 82 Eagle's medium (DMEM, Life Technologies, Grand Island, NY, USA) supplemented with 10\% 83 fetal bovine serum (FBS) and antibiotics (Gibco-BRL/Invitrogen, Carlsbad, CA, USA). Viruses 84 used for this study were foot-and-mouth disease virus (FMDV) isolates of serotype A and 85 vesicular stomatitis Indiana virus (VSV). For FMDV plaque assays, virus dilutions (0.2 ml per 86 well of a 6-well tissue culture plate) were added onto a cell monolayer containing $10^{6}$ BHK-21 
87 cells seeded the day before, and the plates were incubated for $1 \mathrm{~h}$ at $37^{\circ} \mathrm{C}$ to allow virus

88 internalization. Then, the virus inoculum was removed and the cells were overlaid with $2.5 \mathrm{ml}$ of

89 semisolid medium containing SeaPlaque Agarose $0.8 \%$ (Lonza, Rockland, ME, USA) and

90 DMEM supplemented with 2\% FBS. At 48 h postinfection (hpi), cells were fixed with $4 \%$

91 formaldehyde and stained with crystal violet. Plates were washed with tap water, dried and

92 scanned using a flatbed office scanner at 150 or 1200 dots per inch (dpi). For VSV plaque

93 assays, virus dilutions ( $0.1 \mathrm{ml}$ per well of a 24-well tissue culture plate) were added onto a cell

94 monolayer containing $3 \times 10^{5}$ Vero cells seeded the day before, and the plates were incubated for

$951 \mathrm{~h}$ at $37^{\circ} \mathrm{C}$ to allow virus internalization. Then, the virus inoculum was removed and the cells

96 were overlaid with $1 \mathrm{ml}$ of semisolid medium containing methylcellulose $0.7 \%$ and DMEM

97 supplemented with 2\% FBS. At 72 hpi, cells were fixed with 4\% formaldehyde and stained with

98 crystal violet. Plates were washed with tap water, dried and placed over a white background (a

99 white sheet of paper) with the back of the plate facing upwards. Plates were digitalized using a

10013 Megapixels cell phone camera and natural lighting. Direct illumination was avoided (i.e. flash

101 from cell phone was turned off). Alternatively, plates can be placed over a light box illuminator

102 to improve the outline of the plaques.

103

104 Description of ViralPlaque

105 ViralPlaque is written in ImageJ Macro language (IJ), which is a scripting language that allows

106 controlling many aspects of ImageJ (Schindelin et al., 2012). Programs written in this language

107 can be used to perform a desired set of algorithms over the image, which include variables,

108 control structures (conditional or looping statements) and user-defined functions. In addition, the 
109 IJ allows access to all available ImageJ functions and to a vast number of built-in functions.

110 ViralPlaque is available for download in https://sourceforge.net/projects/viralplaque/.

112 An outline of ViralPlaque usage is illustrated in Figure 1. Once the macro is installed, the basic 113 workflow is to open an image file and then run ViralPlaque (Figure 1A). It will ask for specific 114 parameters, method and mode to run and then it performs a set of predefined steps over a user115 defined area of the image being analyzed. The macro includes three methods of image analysis, 116 namely LowRes, Difference, and Weka. The LowRes method was developed to work on low 117 resolution images digitalized using a scanner set at low dpi or simply obtained with a cell phone 118 camera. The other two methods (Difference and Weka) were designed specifically for high 119 resolution images (1200 dpi) obtained from a flatbed scanner. Regarding the running mode, 120 ViralPlaque includes two modes (single well and 6-well). The former (recommended) requires

121 the user to select the area of a culture plate to be analyzed. In case the plaque assay is performed 122 in 6-well plates and the whole plate is digitalized, the user may select the 6-well mode to 123 increase analysis throughput (only available for LowRes and Difference methods).

124 An alternative macro, the Viral Plaque-Batch macro (Figure 1B) allows the user to reuse the 125 functionality of the software on more than one image, increasing throughput even more. Once 126 the batch macro is run, the user is asked to indicate both input and output directories. Then, the 127 ViralPlaque macro is run sequentially on every image of the input directory (the user can change 128 run parameters each time). Finally, once measurements are performed, a results file is 129 automatically saved in the output directory for each image processed.

130 Specific instructions for installing and running both macros including step-by-step overview for 131 each method and mode are listed in Supplementary file 1. 
133 Implementation of ViralPlaque

134 A step-by-step description of the methods included in ViralPlaque is depicted in Figure 2. The

135 LowRes method is the fastest one and includes a set of 5 major steps (Figure 2A). Firstly, two

136 filters are applied to the image, namely Median filter and Gaussian Blur filter. The user is

137 prompted to choose the radius (in pixels) for each filter. Of note, the radius for the Median filter

138 should not be larger than the diameter of the smallest plaque to be detected and the radius for the

139 Gaussian Blur filter should be close to one quarter of the size of the Median radius. Default

140 values were chosen from the images tested in this work; the user should set the values that most

141 fit the input images. Next, thresholding is performed in order to convert the image into black and

142 white. This step can be set to run automatically (default is manual) though this hinders precision.

143 Then, several processes of denoising are performed (erode, dilation, and fill holes) previous to

144 the segmentation step of the watershed command. Watershed is a widespread technique for

145 image segmentation; this step allows the macro to identify as two different objects plaques that

146 are in contact (i.e. merged). Finally, IJ command 'Analyze Particles' is run and the user can

147 control the results obtained (i.e. plaques contours are displayed non-destructively on the

148 duplicate image) manually before proceeding to execute the measure command. Supplementary

149 video 1 exemplifies step-by-step usage of this method. Additionally, if there is no need to obtain

150 size measurements, this method includes a Count Only option where only the number of plaques

151 detected will be informed. This option is designed specifically to count plaques so some

152 parameters will be overwritten to specific values (Supplementary file 1).

153 The Difference method is also a fast method that includes a set of 6 major steps (Figure 2B).

154 First, Image 'Enhance Contrast' function is run, followed by a sharpening process. Users can 
155 change some of these default parameters at the prompt window each time the macro is run. The

156 next step is finding the edges over a duplicate of the image. Then, image calculator is used to

157 create a new image based on the difference between the duplicate and the original image (hence

158 the name of the method). Next, as in the LowRes method, thresholding, denoising and 'Analyze

159 Particles' steps are performed. Supplementary video 2 exemplifies step by step usage of this

160 method.

161 Lastly, the Weka method consists of roughly similar steps as the Difference method though no

162 manual thresholding is performed (Figure 2B). To circumvent this step, Trainable Weka

163 Segmentation plugin (Arganda-Carreras et al., 2017) was used to train classifiers on example

164 images in order to obtain a classifier file (Supplementary file 1). Alternatively, the user can

165 execute the Trainable Weka Segmentation plugin on its own example images in order to obtain a

166 classifier file. For specific instructions see Supplementary file 1. Additionally, Supplementary

167 video 4 exemplifies step-by-step procedure of this training process. Once the macro is run, it

168 produces a duplicate image that will be classified using the Weka plugin. The image is then

169 converted to black and white followed by denoising, filling holes and segmentation steps.

170 Finally, 'Analyze Particles' command is run and manual control of the results obtained can be

171 done. Supplementary video 3 exemplifies step-by-step usage of this method.

172

173 Results

174 We tested the ability of the ViralPlaque macro to detect and measure viral plaques accurately

175 from low resolution images. To this end, digital images of wells $(n=18)$ recorded using a flatbed

176 scanner (150 dpi) from FMDV plaque assays were used. Firstly, manual measurements of the

177 area of individual plaques $(\mathrm{n}=151)$ were performed with ImageJ's 'draw ellipse' and 'measure' 
178 tools (Analyze-Measure) on the original images. Then, ViralPlaque macro was used to measure

179 the area of individual plaques using the LowRes method with default parameters in single well 180 mode (Figure 3A). All false positive plaques (i.e., plaques assigned by ViralPlaque that did not 181 represent actual lysis plaques) were eliminated manually before the measurement step using the 182 ROI manager as described in Supplementary File 1. In some cases, plaques were not detected 183 automatically by the IJ macro (for example, see plaque G in Figure 3A); in other cases, two 184 adjacent plaques were erroneously considered as a single plaque (see plaque $\mathrm{C}$ in Figure $3 \mathrm{~A}$ and 185 Supplementary Figure 1B). Nonetheless, 129 of the manually detected plaques were identified 186 using the automated method, which gives a recall of 0.854 . Moreover, the 10 th and 90 th 187 percentile values of the plaque area proved to be close to those obtained for the manual 188 measurements, suggesting good reliability (Figure 3B and Supplementary Figure 1A). Indeed, a 189 good linear correlation was observed between manual and automated analysis $\left(\mathrm{R}^{2}=0.831\right)$, with a 190 slope value of 1.17 as represented in Figure 3C.

191 In order to test the versatility of ViralPlaque, we tested the macro on images of VSV plaque 192 assays recorded with a cell phone camera. In this case, plaque assays were performed on 24-well 193 culture plates and using a methylcellulose overlay. Manual measurements of the area of 194 individual plaques $(\mathrm{n}=152)$ were performed and compared to the dimensions of plaques detected 195 by the LowRes method. As indicated in Material and Methods, in this case the radius for the 196 Median and the Gaussian filters were increased in order to better suit the analysis to the image 197 resolution (Figure 3D). As shown in Figure 3E, 134 of the manually detected plaques were 198 identified using the automated software (recall=0.881). Again, plaque area 10th and 90th 199 percentile values were similar to those obtained for the manual measurements (Figure 3E and 
200 Supplementary Figure 1D). As with the FMDV assay, a good linear correlation was observed 201 between manual and automated analysis $\left(\mathrm{R}^{2}=0.794\right)$, with a slope value of 1.018 , Figure $\left.3 \mathrm{~F}\right)$. 202

203 Next, we tested the ViralPlaque macro on high resolution images using both Difference and 204 Weka methods with default parameters in single well mode (Figure 4A,B). Since these image 205 conditions increase sensitivity thus allowing for more false positive results, minimum plaque size 206 was increased to $300 \mathrm{px}^{2}$. Most of the manually detected plaques were identified using the 207 automated software, with recall values of 0.883 and 0.834 for Difference and Weka methods, 208 respectively. Again, plaque area presented a similar distribution independently of the method 209 used, suggesting good reliability (Figure 4C,E). As expected, a very good linear correlation was 210 observed between manual and automated analysis, as represented in Figure 4D (slope $=0.990$, $211 \mathrm{R}^{2}=0.9399$ for manual vs. Difference method; slope $=1.085, \mathrm{R}^{2}=0.9314$ for manual vs. Weka 212 method).

213

214 Remarkably, manual measurements of viral plaques took an average of 100 seconds per image, 215 though this is highly dependent on the number of plaques per well. In turn, the average 216 processing time per image using the macro was 30 seconds for the Weka method and even 15 217 seconds for LowRes and Difference methods, representing a $>3$-fold and 5-fold reduction, 218 respectively. Together, the high reliability, robustness and speed of the ViralPlaque macro 219 support its utility for the automated calculation of viral plaque dimensions.

220

\section{Discussion}


223 Imaging programs ideally should be flexible with tunable parameters defined by the user and 224 cost free. Having this in mind, we chose Fiji as a platform, which is a distribution of the popular 225 open-source Java-based image processing program ImageJ focused on biological image analysis 226 (Schindelin et al., 2012). Fiji combines powerful software libraries with a broad range of 227 scripting languages which, in turn, enables rapid implementation of image-processing algorithms 228 and extensive plugins and macros for specific purposes. In this sense, ViralPlaque implements 229 this powerful tool to facilitate a tedious task. Also, the macro includes a prompt stage where the 230 majority of the relevant parameters (including circularity, particle size, enhance contrast, set 231 measurement) can be changed by the user to better fit its needs. Also, if the scale of the image is 232 known, the ratio of pixels per mm can be easily given at this stage, thus prompting the results to 233 be informed in millimeters. Even so, if more specific changes were needed, the macro developed 234 can be easily modified to fit the user's specific requirements. In line with this, as part of the 235 ImageJ software, Fiji has a large and hands-on user community that could aid improving 236 ViralPlaque.

238 Admittedly, machine learning is a fast-growing method of data analysis that automates analytical 239 model building. It is a branch of artificial intelligence based on the idea that systems can learn 240 from data, identify patterns and make decisions with minimal human intervention. In this sense, 241 we applied a previously developed plugin (Arganda-Carreras et al., 2017) to train classifiers that 242 could sort pixels of the image between two classes: cells and background (lytic plaque). The 243 classifier file obtained is called upon by the macro to accurately detect and measure the viral 244 plaques. Moreover, the user has the potential to train their own classifiers, adjusting the macro 245 for the specific conditions of the experiment at hand, thus severely improving performance. 
246 Nonetheless, it should be kept in mind that the Weka method is computationally expensive thus

247 its use is highly dependent on the hardware capabilities at disposal. On the other hand, LowRes

248 and Difference methods are much simpler algorithms (and thus much faster) making them more

249 suitable for most every day computer hardware. It should be noted that those methods are, by

250 default, run with auto-thresholding option off, this means that user input is needed to threshold

251 each image (e.g. selecting the corresponding values of pixels that better separate cells from

252 plaques). Alternatively, this option can be set on, hence reducing user intervention (more

253 reproducibility) but heavily increasing false positive and false negative rates depending on

254 particular image conditions (i.e. illumination, artifacts, contrast).

255

256 Additionally, a batch-mode macro is also presented. Once the user has reached the best

257 parameters and method for the images to be processed, this macro is an alternative that should

258 help increasing speed even further. This is achieved by eliminating repetitive steps such as

259 saving results files and by automatically opening all image files in a particular folder.

260

261 Conclusions

262 ViralPlaque is a fast, open-source, accurate, tunable, and user-friendly image analysis method for

263 the obtention of viral plaques statistics that highly replicates manual measuring and facilitates

264 characterization of cytolytic viruses.

265

266 Acknowledgements

267 M.C. is a fellow of the ANPCyT and A.C. is a doctoral fellow of the National Research Council

268 (CONICET) at the University of Luján, Argentina. M.I.G. is member of CONICET Research 
269 Career Program. We thank Sandra Cordo, María Cruz Miraglia and Sabrina Amalfi for kindly

270 providing images and Matías Richetta for helpful discussions.

271

272

273

\section{References}

275 Arganda-Carreras I, Kaynig V, Rueden C, Eliceiri KW, Schindelin J, Cardona A, Seung HS.

276 2017. Trainable Weka Segmentation: a machine learning tool for microscopy pixel

277 classification. Bioinformatics 33: 2424-2426. DOI: 10.1093/bioinformatics/btx180.

278 Baer A, Kehn-Hall K. 2014. Viral concentration determination through plaque assays: using

279 traditional and novel overlay systems. J. Vis. Exp. (93), e52065. DOI:10.3791/52065.

280 Cai Z, Chattopadhyay N, Liu WJ, Chan C, Pignol JP, Reilly RM. 2011.

281 Optimized digital counting colonies of clonogenic assays using ImageJ software and customized 282 macros: comparison with manual counting. Int J Radiat Biol. 87:1135-46. DOI:

$28310.3109 / 09553002.2011 .622033$.

284 Choudhry P. 2016. High-throughput method for automated colony and cell counting by digital 285 image analysis based on edge detection. PLoS One. 11:e0148469. DOI:

286 10.1371/journal.pone.0148469.

287 Culley S, Towers GJ, Selwood DL, Henriques R, Grove J. 2016. Infection counter: automated 288 quantification of in vitro virus replication by fluorescence microscopy. Viruses 8(7). pii: E201. 289 DOI: 10.3390/v8070201.

290 d'Hérelle F, Smith GH. 1926. The bacteriophage and its behavior. Baltimore: The Williams \& 291 Wilkins Company. 
292 Dulbecco R. 1952. Production of plaques in monolayer tissue cultures by single particles of an 293 animal virus. Proc Natl Acad Sci USA 38:747-752.

294 Fan Q, Kopp SJ, Byskosh NC, Connolly SA, Longnecker R. 2018. Natural selection of 295 glycoprotein B mutations that rescue the small-plaque phenotype of a fusion-impaired herpes 296 simplex virus mutant. Mol. Bio. 9(5). pii: e01948-18. DOI: 10.1128/mBio.01948-18.

297 García Núñez MS, König G, Berinstein A, Carrillo E. 2010. Differences in the virulence of two 298 strains of Foot-and-Mouth Disease Virus Serotype A with the same spatiotemporal distribution. 299 Virus Res. 147:149-52. DOI: 10.1016/j.virusres.2009.10.013.

300 Geissmann Q. 2013. OpenCFU, a new free and open-

301 source software to count cell colonies and other circular objects. PLoS One. 8(2):e54072. DOI:

302 10.1371/journal.pone.0054072.

303 Goh KC, Tang CK, Norton DC, Gan ES, Tan HC, Sun B, Syenina A, Yousuf A, Ong XM, 304 Kamaraj US, Cheung YB, Gubler DJ, Davidson A, St John AL, Sessions OM, Ooi EE. 2016. 305 Molecular determinants of plaque size as an indicator of dengue virus attenuation. Sci Rep. 306 6:26100. DOI: 10.1038/srep26100.

307 Helmy IM, Azim AM. 2012. Efficacy of ImageJ in the assessment of apoptosis. Diagn Pathol. 308 7:15. DOI: 10.1186/1746-1596-7-15.

309 Kato F, Tajima S, Nakayama E, Kawai Y, Taniguchi S, Shibasaki K, Taira M, Maeki T, Lim 310 CK, Takasaki T, Saijo M. 2017. Characterization of large and small-plaque variants in 311 the Zika virus clinical isolateZIKV/Hu/S36/Chiba/2016. Sci. Rep. 7:16160. doi: 10.1038/s41598312 017-16475-2.Katzelnick LC, Coello Escoto A, McElvany BD, Chávez C, Salje H, Luo 313 W, Rodriguez-Barraquer I, Jarman R, Durbin AP, Diehl SA, Smith DJ, Whitehead 314 SS, Cummings DAT. 2018. Viridot: An automated virus plaque (immunofocus) counter for the 
315 measurement of serological neutralizing responses with application to dengue virus. PLoS Negl 316 Trop Dis. 12:e0006862. DOI: 10.1371/journal.pntd.0006862. Moser LA, Boylan BT, Moreira

317 FR, Myers LJ, Svenson EL, Fedorova NB, Pickett BE, Bernard KA. 2018. Growth and

318 adaptation of Zika virus in mammalian and mosquito cells. PLoS Negl Trop Dis. 12: e0006880.

319 DOI: 10.1371/journal.pntd.0006880. Schade-Weskott ML, van Schalkwyk A, Koekemoer JJO.

320 2018. A correlation between capsid protein VP2 and the plaque morphology of African horse

321 sickness virus in cell culture. Virus Genes. 54:527-535. DOI: 10.1007/s11262-018-1567-y.

322 Schindelin J, Arganda-Carreras I, Frise E, Kaynig V, Longair M, Pietzsch T, Preibisch

323 S, Rueden C, Saalfeld S, Schmid B, Tinevez JY, White DJ, Hartenstein V, Eliceiri K, Tomancak

324 P, Cardona A. 2012. Fiji: an open-source platform for biological-image analysis. Nat Methods

325 9:676-82. DOI: 10.1038/nmeth.2019.

326 Sullivan K, Kloess J, Qian C, Bell D, Hay A, Lin YP, Gu Y. 2012. High throughput virus plaque

327 quantitation using a flatbed scanner. $J$ Virol Methods. 179:81-89. DOI:

328 10.1016/j.jviromet.2011.10.003.Tajima S, Nerome R, Nukui Y, Kato F, Takasaki T, Kurane I.

329 2010. A single mutation in the Japanese encephalitis virus E protein (S123R) increases its

330 growth rate in mouse neuroblastoma cells and its pathogenicity in mice. Virology 396:298-

331 304.DOI: 10.1016/j.virol.2009.10.035.

332 Yakimovich A, Andriasyan V, Witte R, Wang IH, Prasad V, Suomalainen M, Greber UF. 2015.

333 Plaque2.0-A high-throughput analysis framework to score virus-cell transmission and clonal

334 Cell expansion. PLoS One 10:e138760. doi: 10.1371/journal.pone.0138760. 
Figure 1

Basic workflow for ViralPlaque (A) and Viral Plaque-Batch (B) macros.

Blue filling color indicates that the step requires user input.

A

ImageJ ViralPlaque macro

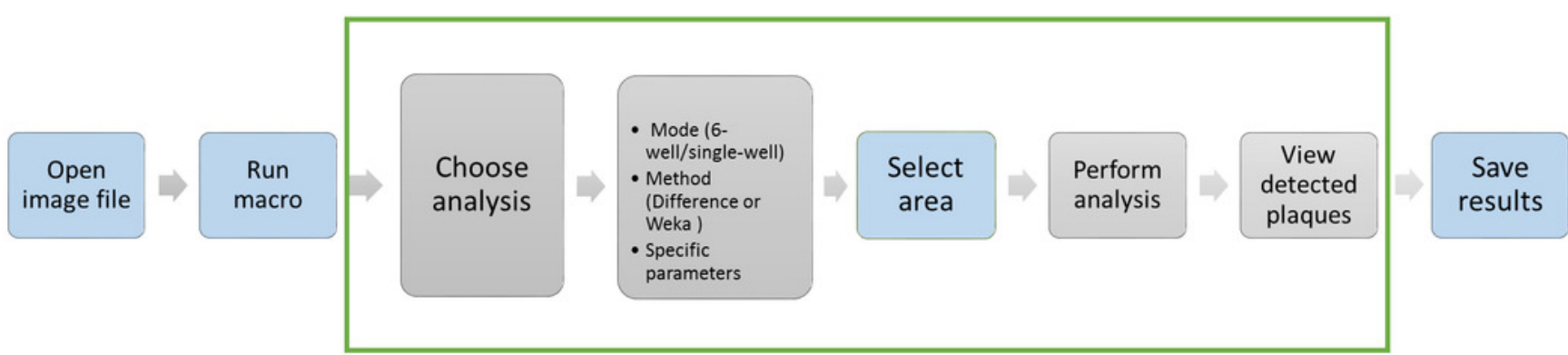

B

ImageJ ViralPlaque-Batch macro

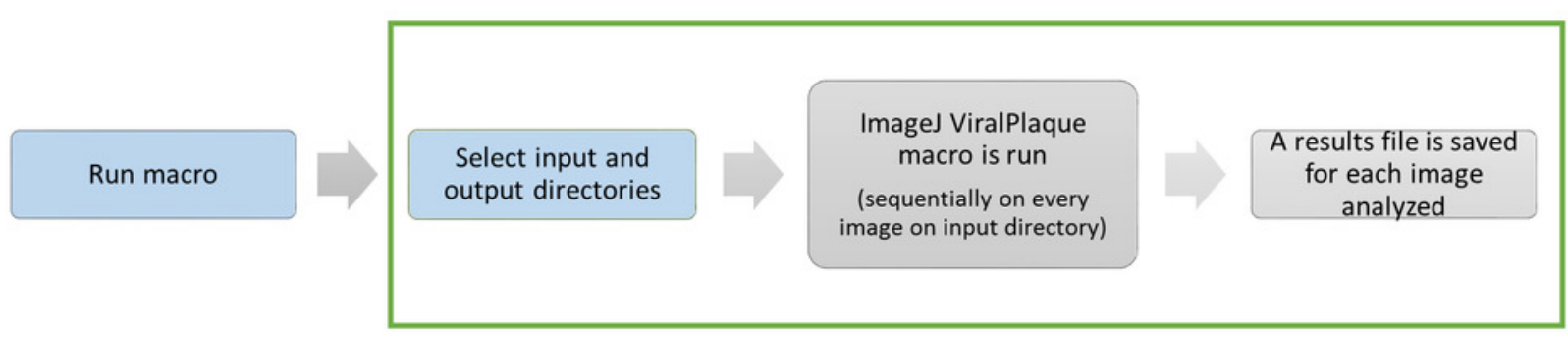


Figure 2

Summary of the processing steps for the three alternative methods included in ViralPlaque macro.

Filling color indicates the condition of each step, where blue: manual or automated modes available (default is manual), green: optional step, orange: parameters can be set at the initial prompt. ROI: region of interest.

A

\section{Low resolution}

LowRes

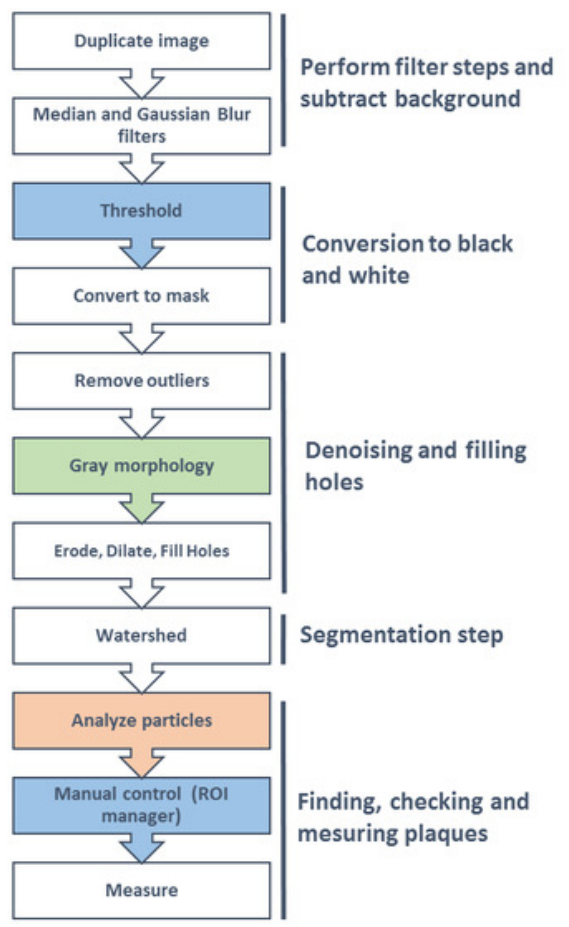

B

High resolution

Difference

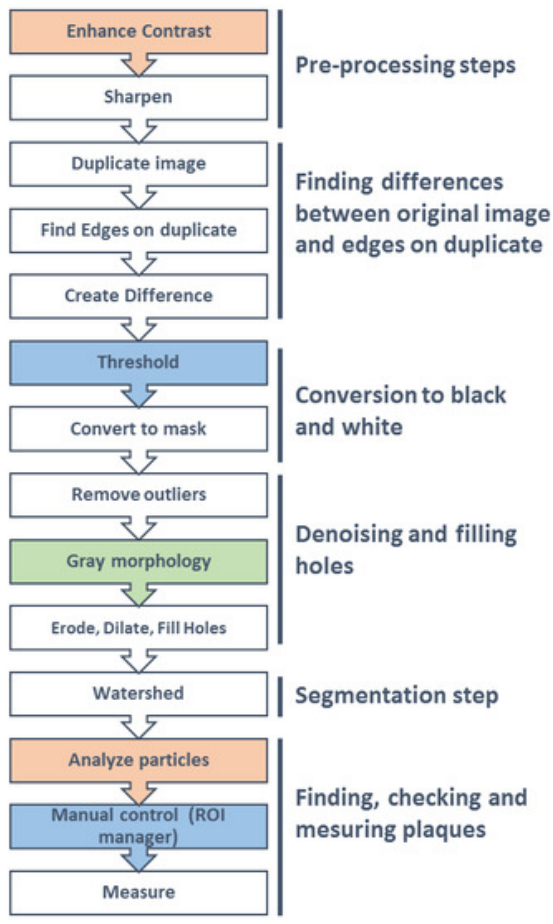

Trainable Weka (machine learning)

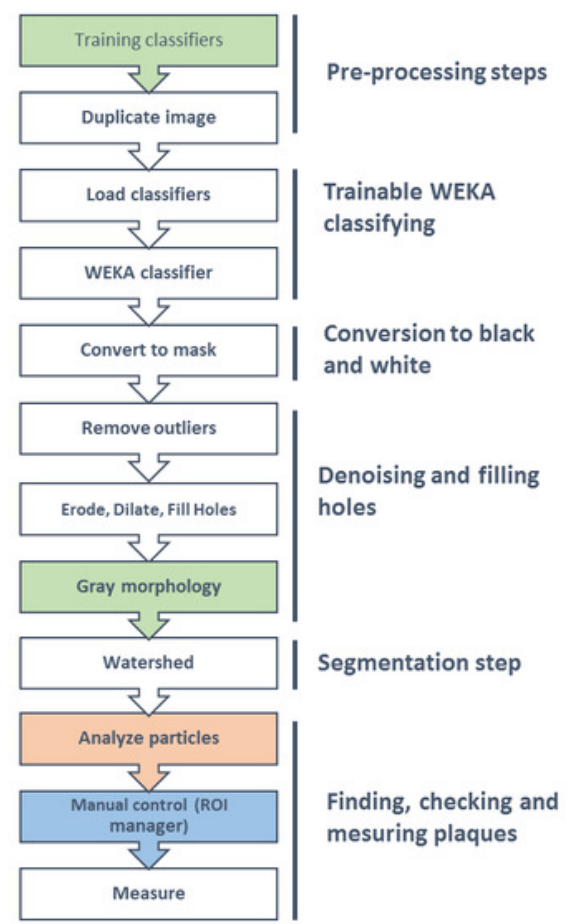




\section{Figure 3}

Comparison of plaque measurements with manual and LowRes methods.

(A-D) Analysis of 6-well FMDV plaque assays scanned using a flatbed office scanner at 150 dpi. (E-H) Analysis of 24-well VSV plaque assays digitalized using a 13 Megapixels cell phone camera. Median filter radius was increased to $8 \mathrm{px}$ and Gaussian Blur filter radius was set at $2 \mathrm{px}$. The original low-resolution images $(A, E)$ were processed with the ViralPlaque macro ( $B$, F). The plaques detected by the macro are circled in yellow and labeled (B,F). In (B), arrows indicate plaques that were only detected manually ( $G$ and $H$ ). (C, G) Distribution of plaque areas. Box-plots represent data obtained between $10^{\text {th }}$ and $90^{\text {th }}$ percentiles; median area is indicated by a horizontal line. The number of plaques detected by each method is given in brackets. (D, H) Correlation between manual and digital measurements of individual plaque area. The equation of the linear least-squared fit and goodness of fit $R^{2}$ is given for each method. 


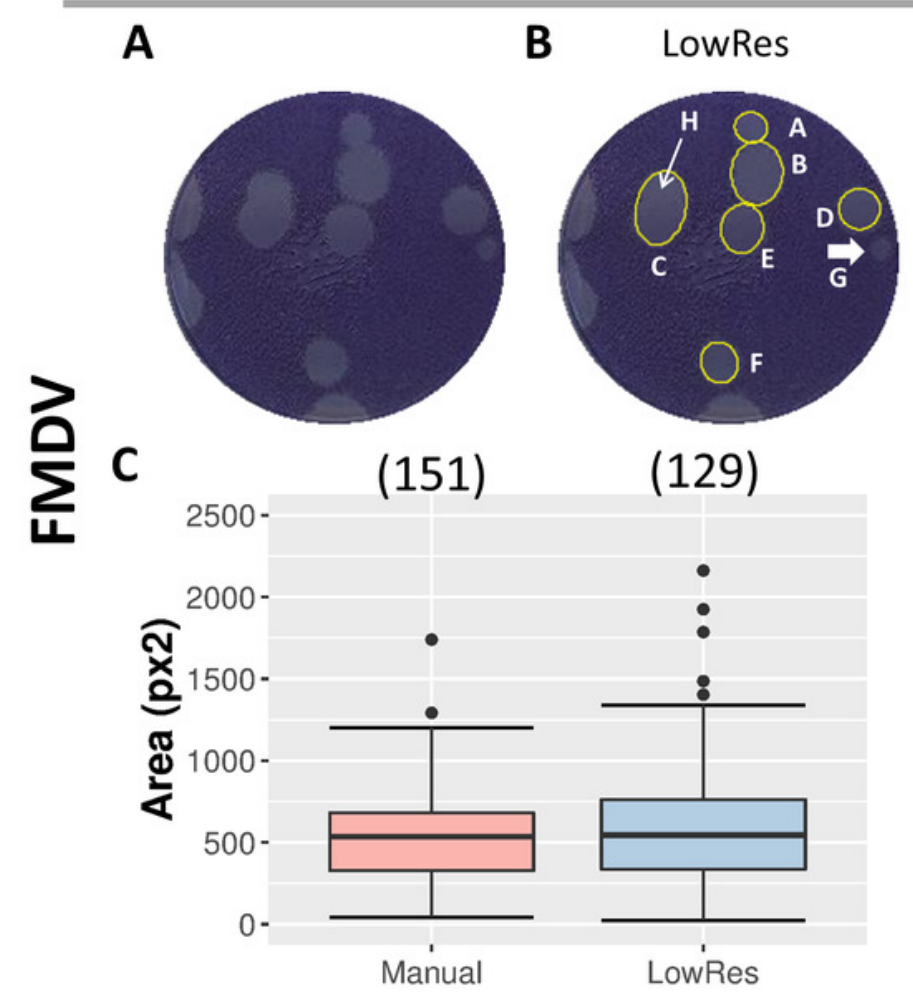

D
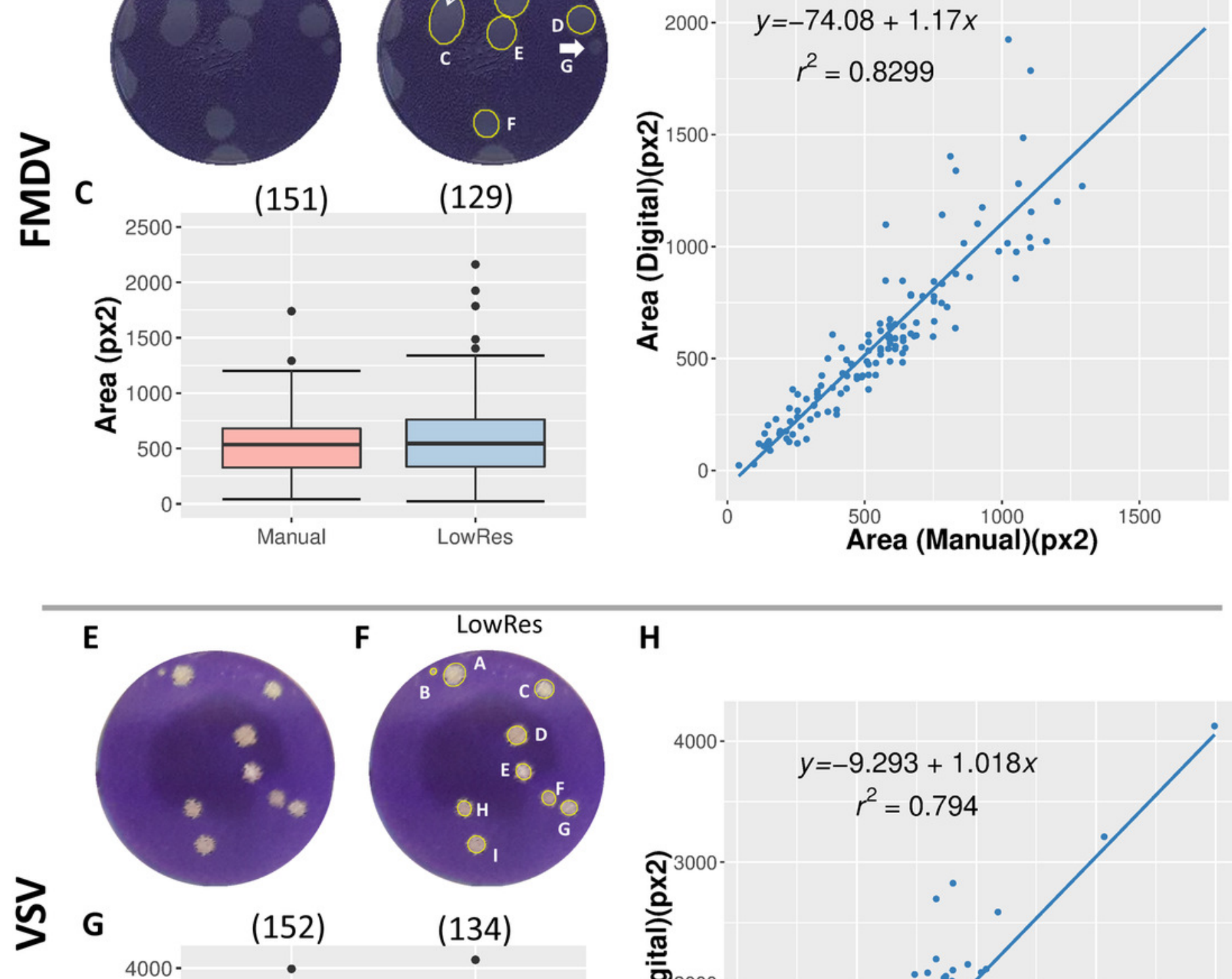

H

4000 -

$$
\begin{gathered}
y=-9.293+1.018 x \\
r^{2}=0.794
\end{gathered}
$$

(134)
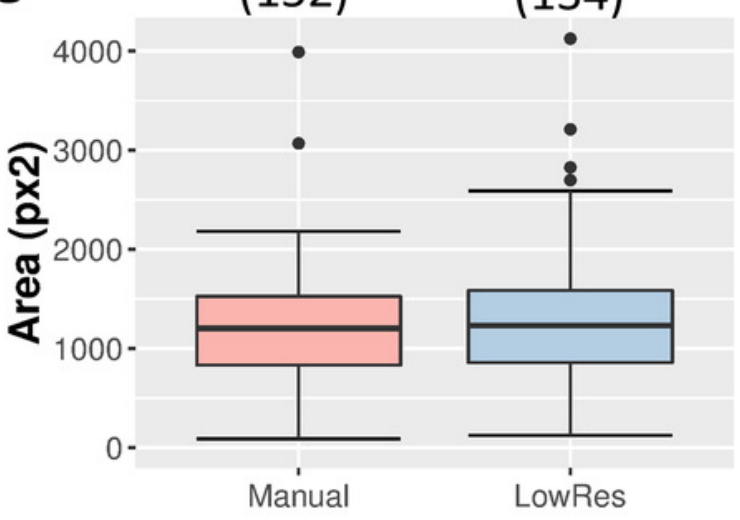

กั้

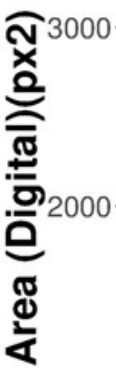

1000 -

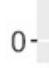

i

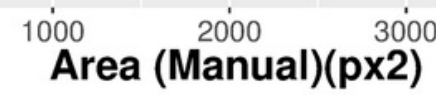




\section{Figure 4}

Comparison of Difference and Weka methods with manual measurements.

Analysis of 6-well FMDV plaque assays scanned using a flatbed office scanner at $1200 \mathrm{dpi}$. High-resolution original image (A) and images processed by the manual method (B) and by If macro, Difference $(C)$ and Weka $(D)$ methods. The plaques detected by the macro are circled in yellow and labeled from $A$ to $F$. (E) Area of the 6 plaques detected in (A) as determined by Manual, Difference and Weka methods. (F) Distribution of plaque areas of the total number of plaques analyzed from 17 images. Box-plots represent data obtained between $10^{\text {th }}$ and $90^{\text {th }}$ percentiles; median area is indicated by a horizontal line. The number of plaques detected by each method is given in brackets. (G) Correlation between manual and digital measurements of the area of individual plaques. The equation of the linear least-squared fit and goodness of fit $\mathrm{R}^{2}$ is given for each method. (H) Distribution of plaque areas from 17 images measured manually and using ViralPlaque macro methods. Boxes represent distribution of data between $10^{\text {th }}$ and $90^{\text {th }}$ percentile; horizontal lines indicate median values. 
A

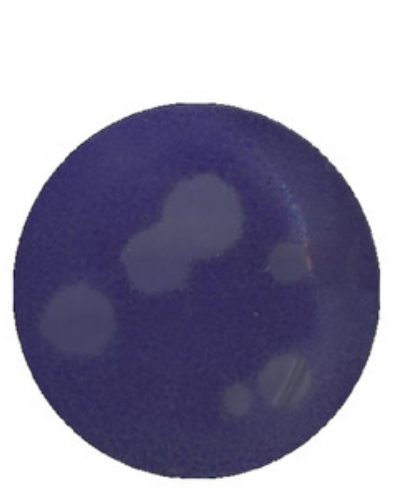

$E$

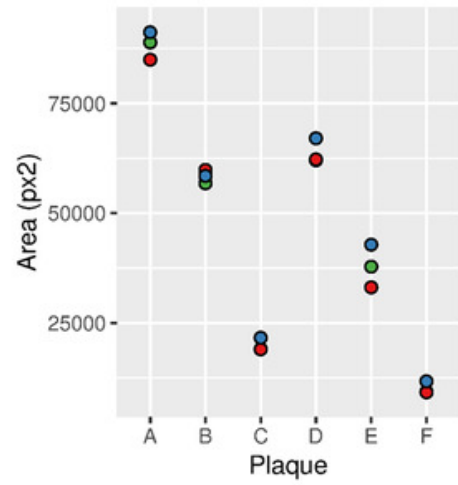

$\mathrm{F}$

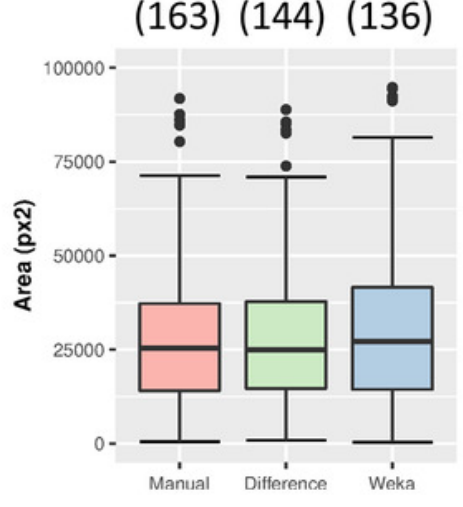

B

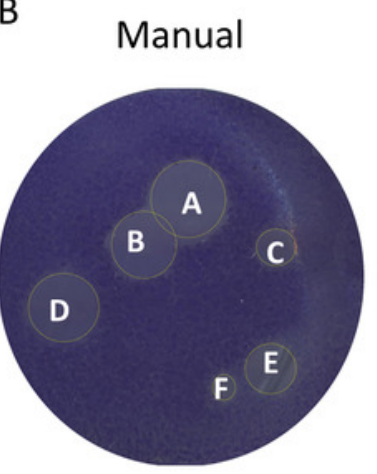

C

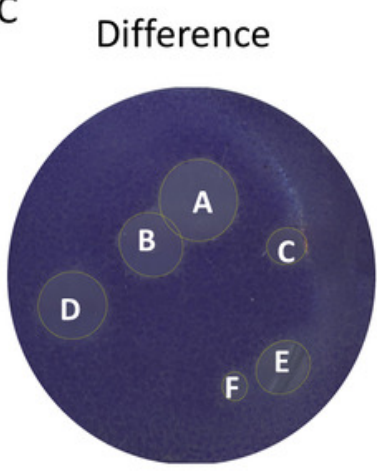

D Weka

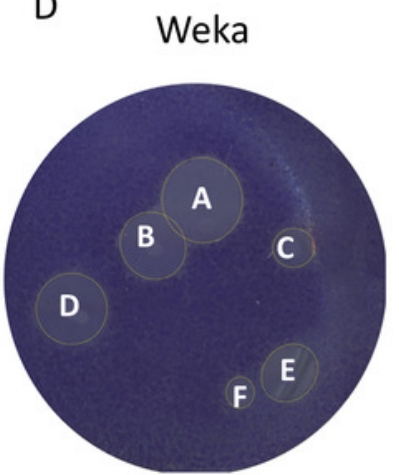

G

O Difference

- Manual

- Weka

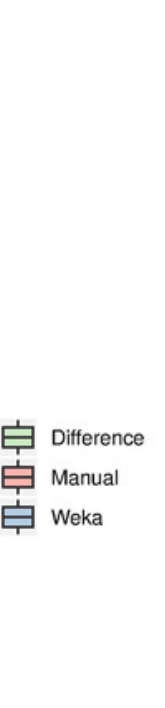

$100000-$

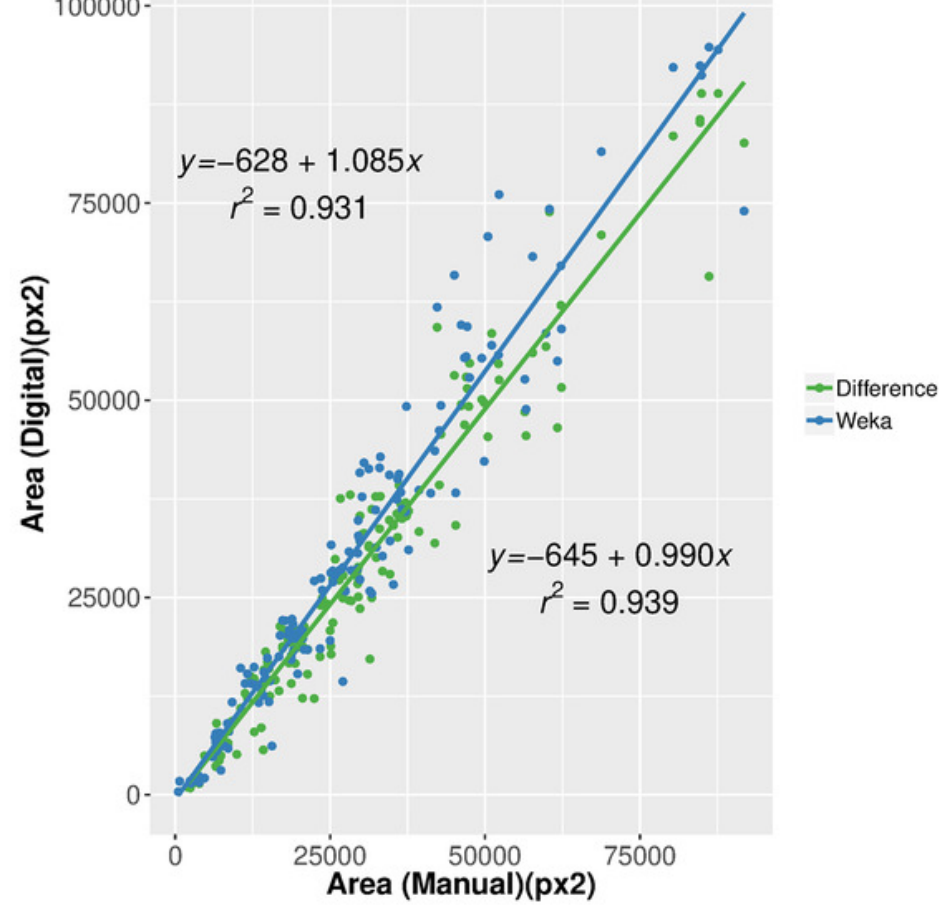

$\mathrm{H}$

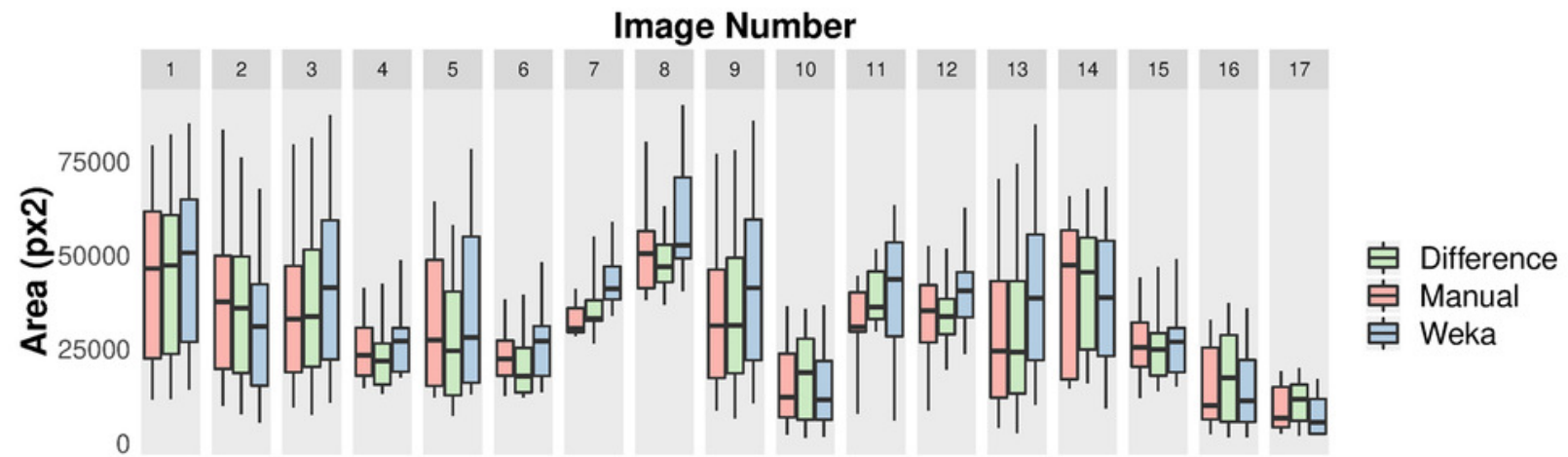

